\title{
Eficiencia reproductiva y producción de polen en Nothofagus alpina en un huerto semillero clonal
}

\author{
Reproductive efficiency and pollen production in a Nothofagus alpina clonal seed orchard
}

\author{
Luz García Cruzatty abc*, Magaly Rivero ${ }^{\mathrm{d}}$, Gregorio Vásconez ${ }^{\text {b}}$, Soraya Peñarrieta ${ }^{\mathrm{c}}$, Fernando Droppelmann ${ }^{\mathrm{e}}$ \\ * Autor de correspondencia: ${ }^{a}$ Universidad Austral de Chile, Facultad de Ciencias Forestales y Recursos Naturales, \\ Programa de Doctorado en Ciencias Forestales, Valdivia, Chile, luzcecilia29@hotmail.com

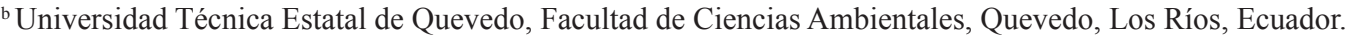 \\ ' Universidad Técnica de Manabí, Facultad de Ingeniería Agronómica, Campus Experimental La Teodomira, \\ km 13,5 Vía a Santa Ana, Santa Ana, Manabí, Ecuador. \\ ${ }^{\mathrm{d}}$ Cooperativa de Mejoramiento Genético de Chile, Valdivia, Chile. \\ ${ }^{\text {e } U n i v e r s i d a d ~ A u s t r a l ~ d e ~ C h i l e, ~ F a c u l t a d ~ d e ~ C i e n c i a s ~ F o r e s t a l e s, ~ I n s t i t u t o ~ d e ~ B o s q u e s ~ y ~ S o c i e d a d, ~ V a l d i v i a, ~ C h i l e . ~}$
}

\begin{abstract}
SUMMARY
The potential production of pollen per plant is a good indicator of fruit production. In this work the reproductive efficiency index, the average potential pollen production per flower and the functional relationship between the number of anthers and the amount of pollen per flower of Nothofagus alpina were determined. The ratio of viable seeds formed after manual pollination and the seeds formed by natural pollination was calculated. The total of pollen grains per flower was estimated by direct counting. The index of reproductive efficiency was estimated at an average of 0.19. It was found that the number of pollen grains per anther varied among individuals and among years. Pollen production per flower was estimated between 85,703 pollen grains per flower in 2011 and 117,975 in 2013. Low seed production after open pollination could be due to pollen limitation, since the formation of viable seeds after hand pollination was significantly higher. The amount of pollen content in the flowers of $N$. alpina varies in a direct relation with the pollen grains in the anthers, suggesting that the final production of pollen does not depend on the number of anthers per flower.
\end{abstract}

Key words: pollen, reproduction, Nothofagaceae, anemophily.

\section{RESUMEN}

El potencial de producción de polen por planta es un buen indicador de la producción de frutos. En este trabajo se calculó el índice de eficiencia reproductiva, el potencial de producción promedio de polen por flor y se determinó la relación funcional entre el número de anteras y la cantidad de polen por flor en Nothofagus alpina. Se calculó la proporción entre las semillas viables formadas después de polinización manual y las formadas mediante polinización natural. El total de granos de polen por flor se estimó por conteo directo. El índice de eficiencia reproductiva se calculó en un promedio de 0,19 . Se encontró que el número de granos de polen por anteras varió entre individuos y entre años. La producción de polen por flor se calculó entre 85.703 granos de polen por flor en el año 2011 y 117.975 en el año 2013. La baja producción de semillas en condiciones de polinización abierta puede deberse a limitación polínica, pues la formación de semillas viables después de polinización manual fue notablemente mayor. La cantidad de polen contenido en las flores de $N$. alpina varía en relación constante con los granos de polen en las anteras, lo que sugiere que la producción final de polen no depende de la modificación del número de anteras por flor.

Palabras clave: polen, reproducción, Nothofagaceae, anemofilia.

\section{INTRODUCCIÓN}

La polinización eficiente, y la consiguiente producción de frutos, dependen de la producción de polen y de otros factores, como las distancias entre individuos co-específicos y/o la eficiencia en la dispersión de polen (Allison 1990). En general, la cantidad de polen producido en plantas anemófilas es más alta que la producida por plantas polinizadas por insectos (Tormo et al. 1996). Friedman y Barrett (2009) evidenciaron que la eficiencia de la transferencia de polen en plantas anemófilas no es sustancialmente inferior a la de las plantas polinizadas por animales, hallazgos que desafían la explicación de que la evolución a pocos óvulos en flores polinizadas por el viento se asocia con bajas cargas de polen. Esta investigación pone en duda lo supuesto por mucho tiempo de que la polinización anemófila es menos eficaz que la polinización biótica, falta de eficiencia que se compensaría con la producción de una gran cantidad de granos de polen. 
Nothofagus alpina (P. et E.) es una especie nativa de los bosques templados de Chile y Argentina, cuyo mecanismo anemófilo en la dispersión del polen es favorecido por la anatomía floral. Las flores masculinas, dispuestas en las axilas de las hojas más basales ( 1 a 5 por axila), se encuentran sostenidas por largos pedicelos que facilitan su movimiento con el viento, al igual que una elevada cantidad de estambres por flor que varía entre 34 a 50 unidades. Las flores femeninas se encuentran en inflorescencias constituidas por tres flores, son inconspicuas, con estigmas sésiles, grandes y sobresalientes, levemente mucilaginosos en la fase receptiva (García et al. 2013).

En la actualidad existe una fuerte demanda por material vegetal para la reforestación con esta especie, lo que justifica la realización de investigaciones que permitan conocer sus mecanismos reproductivos y por ende la búsqueda de soluciones para mejorar la productividad y calidad de las semillas (García et al. 2014). El conocimiento del potencial de producción de polen por planta y de los periodos de producción es un buen indicador de la salud de los bosques y especialmente es útil en huertos dedicados a la producción de semillas o frutos.

La mayoría de las teorías sobre las estrategias de asignación sexual de las plantas asumen que la disponibilidad de polen normalmente no limita la producción de semillas en las poblaciones de plantas (Burley y Willson 1983). El efecto de la limitación del polen en la producción de semillas se ha observado en varias especies (Bierzychudek 1981, Ashman et al. 2004, Pearse et al. 2015); sin embargo, no existen publicaciones sobre la producción de polen de $N$. alpina, y menos aún de la relación funcional entre el número de anteras y la cantidad de polen por flor.

Los antecedentes sugieren que la eficiencia reproductiva de $N$. alpina no es limitada por una baja disponibilidad de polen, como se ha observado en la mayoría de especies anemófilas (Tormo et al 1996, Piotrowska 2008, GómezCasero et al. 2007), sino más bien a la ineficiencia de este mecanismo de dispersión que resulta en una escasa cantidad de polen que llega a los estigmas (García et al. 2014). Además, de una temporada a otra, la variación observada en la producción de polen por flor no se asocia a la reducción del número de anteras, tal como se ha sugerido al observarse que el número de anteras por flor tiene un amplio rango de variación en $N$. alpina ( 34 - 50 unidades) (García et al. 2013).

Con estas referencias se plantearon como objetivos: calcular el índice de eficiencia reproductiva, estimar el potencial de producción promedio de polen por flor que puede producir un individuo de $N$. alpina, y determinar la relación funcional entre el número de anteras y la cantidad de polen por flor.

\section{MÉTODOS}

El estudio se realizó en el huerto semillero clonal "Huillilemu" de N. alpina, localizado en la comuna San José de la Mariquina, región de Los Ríos. Fue establecido en el año 1989 por la Corporación Nacional Forestal de Chile y la Cooperativa de Mejoramiento Genético Forestal con el objetivo de conservar la diversidad genética de $N$. alpina y su utilización en programas de mejoramiento. Se encuentra fuera del área de distribución natural de la especie, entre las coordenadas geográficas: $39^{\circ} 32^{\prime} 56,57^{\prime \prime}$; $73^{\circ} 04^{\prime} 18,15^{\prime}$ 'O. Este huerto semillero, está constituido por individuos de $N$. alpina originarios de un amplio rango altitudinal en la cordillera de Los Andes, comuna Panguipulli. Dichos árboles tienen aproximadamente la misma edad y están bajo las mismas condiciones ambientales. El huerto tiene una superficie de 3,1 hectáreas y un total de 33 clones, establecidos a un espaciamiento de $5 \times 5 \mathrm{~m}$.

La oscilación térmica mensual en esta localidad, durante los años en que se realizó este estudio, estuvo entre máximas de $17,8(2013)$ a $18,8^{\circ} \mathrm{C}$ (2012) en enero, y mínimas de 5,3 (2011) a $6,5^{\circ} \mathrm{C}$ (2012) en julio. La mayor precipitación se dio durante junio del $2012(337,2 \mathrm{~mm})$, en contraste con el mes febrero del 2011 que tuvo la más baja precipitación (15,4 mm) (figura 1) (Servicio de Meteorología de Chile 2016).

Para determinar el índice de eficiencia reproductiva (IER), se realizaron ensayos de cruzamientos controlados, durante la temporada de floración 2012 (septiembre). Se realizó polinización manual en flores que habían sido embolsadas en la fase de yema para evitar la polinización natural. Previamente se habían etiquetado y aislado ramas en cada uno de los cinco clones seleccionados. La polinización cruzada manual se realizó colocando polen fresco de individuos colindantes sobre los estigmas receptivos de las inflorescencias. Se utilizaron ramas, cada una de las cuales tenían entre 15 y 23 inflorescencias.

Para determinar la producción natural de semillas, antes de la dehiscencia de las infrutescencias, se colocaron mallas en 30 ramas en diferentes orientaciones, en los mismos clones en que se hizo la polinización cruzada manual. En la época adecuada se tomaron las semillas obtenidas en cada árbol seleccionado y se separaron manualmente las llenas de las vacías.

Se determinó el índice de eficiencia reproductiva (IER), calculando el cociente entre la producción de semillas en inflorescencias de polinización abierta y la producción de semillas después de polinización cruzada manual (Ruiz- Zapata y Arroyo 1978). Para facilitar la redacción de la metodología y de los resultados se consideró que un fruto corresponde a una semilla, pues el fruto de $N$. alpina es monospermo, tipo nuez. Los promedios del IER para cinco clones (un rameto por clon) fueron contrastados mediante una comparación múltiple, utilizando la prueba no paramétrica de Kruskal-Wallis.

El total de granos de polen por flor se estimó por conteo directo, siguiendo la metodología de Shivanna y Rangaswamy (1992): se colectaron al azar, aproximadamente a 1,50 m de altura de la copa, 20 flores por tres clones codificados como 11, 23 y 30 (un rameto por clon), du- 


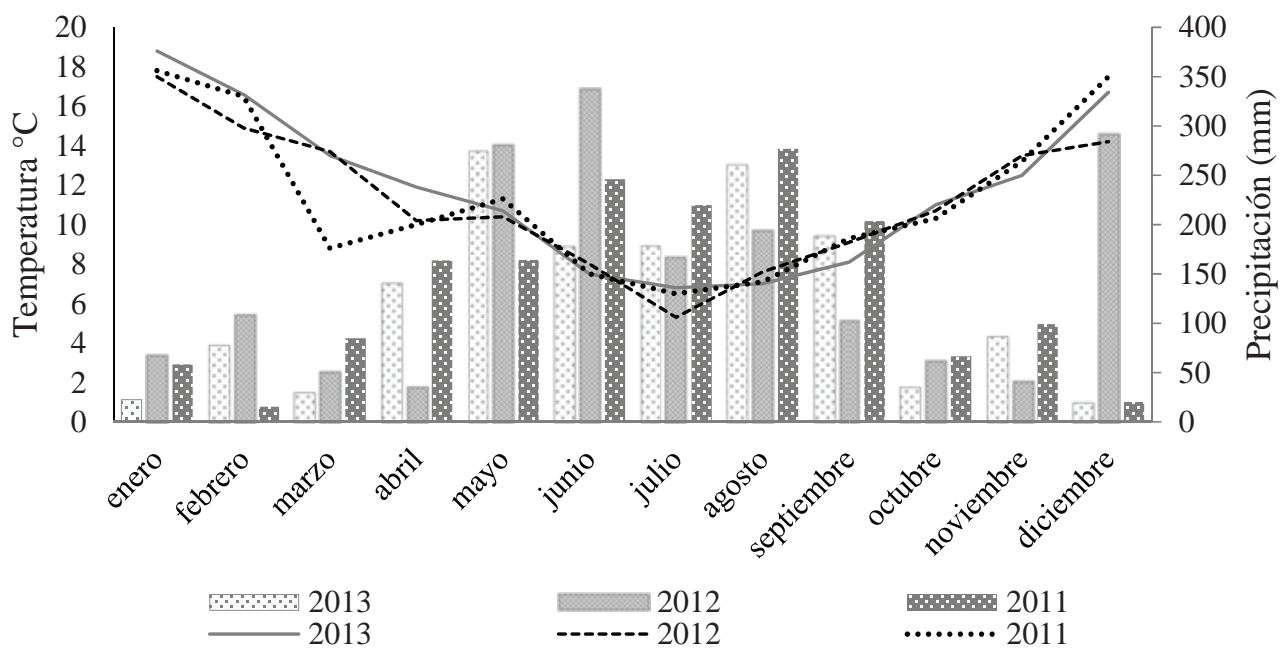

Figura 1. Precipitación (barras) y temperatura media mensual (líneas) durante el periodo de estudio en el huerto semillero "Huillilemu". Climatic conditions in the period of study in the seed orchard "Huillilemu". Precipitation (bar) and temperature (lines).

rante tres años consecutivos $(2011,2012,2013)$; dichas flores se mantuvieron en FAA (formaldehido, alcohol, ácido acético) a $4{ }^{\circ} \mathrm{C}$ hasta su utilización. De cada flor se tomaron cinco anteras, las que fueron colocadas en tubos de ensayo con $200 \mu \mathrm{L}$ de agua y azul de anilina en lactofenol y aplastadas con una varilla de vidrio. El total de granos de polen se contó desde esta preparación, utilizando un hemocitómetro (marca Marienfeld, modelo 0610130). Por último, el número de granos de polen por anteras (GPA) fue determinado utilizando la fórmula 1, y luego este valor fue multiplicado por el total de anteras de cada flor.

$G P A=\frac{\text { Granos de polen en suspensión (volumen total de la suspensión) }}{\text { Volumen de la suspensión usada para el conteo }}$

Para el análisis de los componentes determinantes (anteras flor $^{-1}$, polen antera ${ }^{-1}$, polen flor ${ }^{-1}$ ), los niveles del factor clon y el factor año fueron ordenados de manera factorial y distribuidos en un diseño completamente al azar con 20 repeticiones (20 flores). La significancia de los factores, y su interacción se estableció a través del nivel de probabilidad derivados del análisis de varianza. Además, las diferencias entre los niveles de cada factor se establecieron a través de la prueba de Tukey con un nivel de probabilidad de 0,05 . Por otro lado, se realizó un análisis de regresión entre el número de anteras por flor y el número de gránulos por antera con respecto al contenido total de polen por flor. La significancia de la relación entre los factores determinantes de la producción de polen se valoró a través del coeficiente de determinación $\left(\mathrm{R}^{2}\right)$ y el valor crítico de Pearson a un nivel de 0,01 . Estos análisis se realizaron utilizando el software Statistica 7.

\section{RESULTADOS}

El índice de eficiencia reproductiva varió entre 9,5 y $37,0 \%$ (cuadro 1). En todos los individuos, las pruebas de polinización cruzada indican que la fertilidad natural en la especie es baja. En cuanto a la formación de semillas de polinización abierta (T1); los valores oscilaron entre 9,0 y $24,9 \%$. Al contrario, la producción de semillas mediante polinización cruzada manual fue superior al $90 \%$ en todos los individuos, a excepción del clon 38 (67,5\%).

La cantidad de granos de polen por antera y por flor fue diferente dentro de un mismo año y entre años $(P<0,0001)$ en un mismo individuo. La producción de polen por antera estuvo en un rango de 2.255 a 2.634 granos. En el individuo 11 se obtuvo el promedio más alto de granos de polen por flor y de número de anteras por flor $(44,6)$ (cuadro 2 ).

$\mathrm{El}$ número de anteras por flor varió entre clones y entre periodos de evaluación. Los clones 11 y 23 presentaron una menor producción de anteras por flor en el año 2013, en relación a los periodos 2011 y 2012; mientras el clon 30 tuvo un similar número de anteras en los años 2011 y 2013, pero inferior en el año 2012 (figura 2A).

Se presentó la menor producción de granos de polen por antera en el año 2011 en los clones 23 y 30; aunque en el año 2013 el clon 23 presentó un aumento notorio en la producción de polen, pasando de menos de 2.000 granos de polen en los periodos 2011 y 2012 a 4.399 granos de polen por antera (figura $2 \mathrm{~B}$ ).

Se encontró una alternancia entre mayor y menor producción de polen en los periodos evaluados. El total calculado de granos de polen por flor estuvo entre $65.469,7$ (clon 30) en el año 2011 y 151.762,6 (clon 23) en el año 2013 (figura 2C). 
Cuadro 1. Formación de semillas en Nothofagus alpina en condiciones de polinización natural (T1) y mediante polinización cruzada manual (T2), e índice de eficiencia reproductiva (IER = T1/ T2).

Formation of Nothofagus alpina seeds under natural pollination (T1) and by manual cross-pollination (T2). Reproductive Efficiency Index (REI) (T1/T2) was calculated.

\begin{tabular}{|c|c|c|c|c|c|c|}
\hline & Clones & $\begin{array}{c}\text { Semillas } \\
\text { colectadas }^{++} \text {(a) }\end{array}$ & $\begin{array}{c}\text { Semillas llenas } \\
\text { (b) }\end{array}$ & $\begin{array}{c}\text { Semillas vacías } \\
(\mathrm{a}-\mathrm{b})\end{array}$ & $\begin{array}{c}\text { Semillas por flor } \\
\text { (b/a) }\end{array}$ & $\begin{array}{c}\mathrm{IER}^{+} \\
(\mathrm{T} 1 / \mathrm{T} 2)\end{array}$ \\
\hline$T 1$ & 11 & 67 & 6 & 61 & 0,090 & \multirow{2}{*}{$0,095 \mathrm{~d}$} \\
\hline$T 2$ & 11 & 151 & 143 & 8 & 0,947 & \\
\hline T1 & 34 & 2.065 & 420 & 1.645 & 0,203 & \multirow{2}{*}{$0,210 \mathrm{~b}$} \\
\hline$T 2$ & 34 & 166 & 161 & 5 & 0,970 & \\
\hline T1 & 36 & 226 & 38 & 188 & 0,168 & \multirow{2}{*}{$0,173 \mathrm{~b}$} \\
\hline$T 2$ & 36 & 178 & 173 & 5 & 0,972 & \\
\hline T1 & 38 & 4.885 & 1.218 & 3.667 & 0,249 & \multirow{2}{*}{$0,370 \mathrm{a}$} \\
\hline$T 2$ & 38 & 206 & 139 & 67 & 0,675 & \\
\hline T1 & 46 & 512 & 55 & 457 & 0,107 & \multirow{2}{*}{$0,114 \mathrm{c}$} \\
\hline$T 2$ & 46 & 186 & 175 & 11 & 0,941 & \\
\hline \multirow{2}{*}{\multicolumn{2}{|c|}{$\begin{array}{l}\text { Promedio } \\
\text { Desviación estándar }\end{array}$}} & & & & & 0,192 \\
\hline & & & & & & 0,109 \\
\hline
\end{tabular}

${ }^{+}$Letras diferentes indican diferencias estadísticas, prueba Kruskal Wallis. ${ }^{++}$Total de semillas colectadas por árbol corresponde al número de flores polinizadas.

Cuadro 2. Promedios ${ }^{+}$y cuadrados medios de los componentes determinantes de la producción de polen en tres clones de Nothofagus alpina y en tres periodos de producción.

Average and mean squares for the key components of pollen production in three Nothofagus alpina clones and three production periods.

\begin{tabular}{|c|c|c|c|c|}
\hline \multirow{2}{*}{ Factor } & \multirow{2}{*}{$\mathrm{gl}$} & \multicolumn{3}{|c|}{ Componentes de la producción de polen de Nothofagus alpina } \\
\hline & & Anteras flor-1 & Polen antera ${ }^{-1}$ & Polen flor ${ }^{-1}$ \\
\hline \multicolumn{5}{|l|}{ Clon } \\
\hline 11 & & $44,6 \mathrm{a}$ & $2.479,2 \mathrm{ab}$ & $111.814 \mathrm{ab}$ \\
\hline 23 & & $42,6 \mathrm{ab}$ & $2.634,5 \mathrm{a}$ & $104.938 \mathrm{ab}$ \\
\hline 30 & & $43,1 \mathrm{~b}$ & $2.255,3 \mathrm{~b}$ & 96.622 b \\
\hline $\bar{x}$ & & 43,43 & $2.456,33$ & 104.46 \\
\hline \multicolumn{5}{|l|}{ Año } \\
\hline 2011 & & 48,1 a & $1.780,0 \quad$ c & \\
\hline 2012 & & $43,7 \mathrm{~b}$ & $2.483,1 \quad b$ & $85.703 \mathrm{~b}$ \\
\hline 2013 & & $38,5 \mathrm{c}$ & $3.105,8$ a & $109.696 \mathrm{a}$ \\
\hline & & & & $117.975 \mathrm{a}$ \\
\hline $\bar{x}$ & & 43,43 & $2.456,33$ & 104.46 \\
\hline Clon & 2 & 64,35 & 2180821,49 & 3472028157,00 \\
\hline Año & 2 & 1367,02 & 26401887,04 & 16857289339,00 \\
\hline Clon x Año & 4 & 563,82 & 17604515,67 & 30452220975,00 \\
\hline Error experimental & 171 & 13,55 & 489130,60 & 967435375,94 \\
\hline Total & 179 & & & \\
\hline Probabilidad $_{\text {(clon) }}$ & & 0,0099 & 0,0130 & 0,0297 \\
\hline Probabilidad $_{(\text {año) }}$ & & $<0,0001$ & $<0,0001$ & $<0,0001$ \\
\hline Probabilidad $_{(\text {clon } x \text { año) }}$ & & $<0,0001$ & $<0,0001$ & $<0,0001$ \\
\hline
\end{tabular}

${ }^{+}$Letras diferentes indican que hay diferencias significativas. 

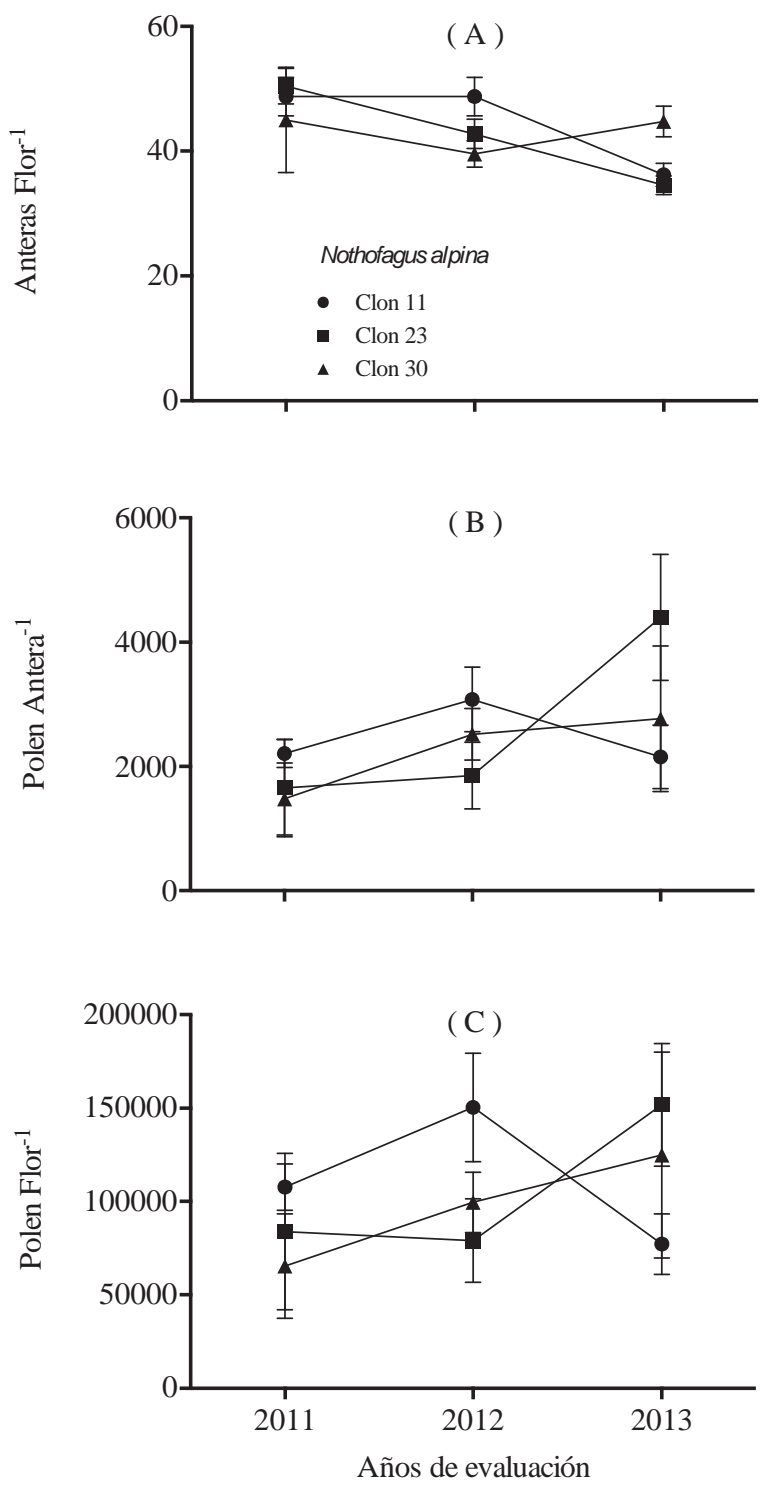

Figura 2. Interacción entre los factores clon y año de evaluación en las anteras por flor (A), polen por antera (B) y polen por flor (C) en Nothofagus alpina.

Clone by year of assessment interaction on anthers per flower (A), pollen per anther (B) and pollen per flower (C) in three Nothofagus alpina clones and three production periods.

En la figura 3A se observa que el número de anteras por flor no determinó la cantidad de granos de polen por flor, ya que no se observó una relación entre estas dos variables. Sin embargo, a pesar de no haberse observado una relación funcional entre el número de anteras por flor y el número de anteras (figura 3C), el número de granos por antera sí determinó la cantidad de granos de polen por flor (figura 3B); observándose, independientemente del clon y del año de evaluación, que por cada grano de polen por antera el aumento en el contenido de granos de polen por flor fue, en promedio, de aproximadamente 42 unidades.
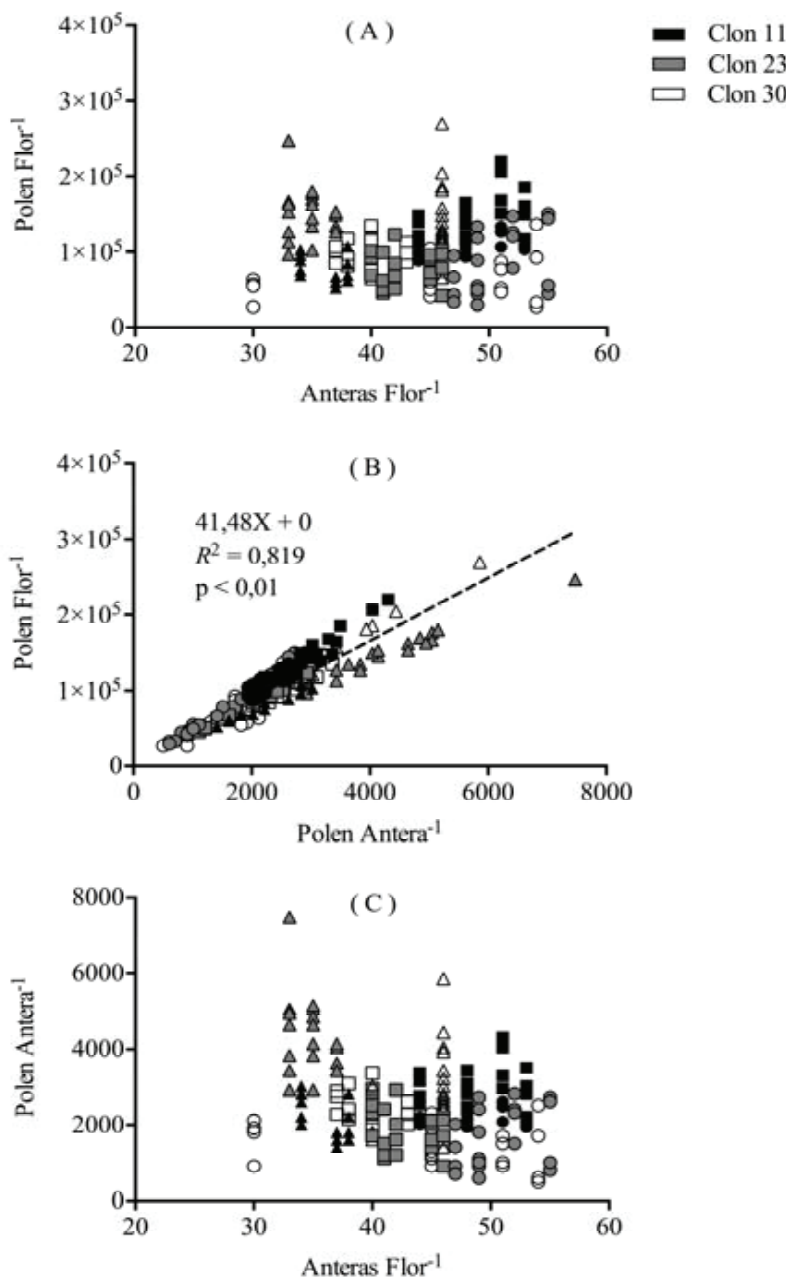

Figura 3. Variación del contenido de polen por flor en función de función de las anteras por flor (A) y el polen por antera (B), y el polen por antera en función de las anteras por flor (C), en tres clones de Nothofagus alpina en tres años de producción. La forma geométrica representa el año: círculo $=2011$, cuadro $=2012$ $\mathrm{y}$ triángulo $=2013 . \mathrm{n}=180$.

Functional relationship among changes in the production of pollen per flower and anthers per flower (A) and pollen per anther (B), and pollen per anther depending on the anthers per flower (C), in three Nothofagus alpina clones and three production periods. The geometric shape represents the year: circle $=2011$, square $=2012$ and triangle $=$ 2013. $\mathrm{n}=180$.

\section{DISCUSIÓN}

Nothofagus alpina presenta mayor producción de semillas llenas después de polinización cruzada manual, en comparación con la producción obtenida mediante polinización abierta, lo que comprueba que la limitación de polen debe ser considerado como un determinante de la baja producción de semillas viables en el huerto "Huillilemu". No obstante, el índice de eficiencia reproductiva varía entre clones, lo que podría deberse a los diferentes índices de 
sobreposición entre las fases de receptividad estigmática y liberación del polen (García et al. 2013); aunque se debería considerar el análisis de esta característica durante varios periodos consecutivos y en una muestra más grande, de esta forma corroborar si los índices de eficiencia reproductiva son característicos para determinados genotipos, por ejemplo, la muy baja proporción de semillas por flor resultado de polinización abierta en el clon 11. En este huerto semillero, el desfase de las fenofases reproductivas entre árboles, limita la polinización cruzada (García et al. 2013), aunque, es posible que en poblaciones naturales, con una mejor sincronía floral entre individuos y por ende un mejor flujo polínico, una mayor cantidad de polen llegue a los estigmas.

Aunque ya se ha mencionado que la limitación de polen sería causada por la asincronía en las fenofases reproductivas entre los diferentes clones del huerto (García et al. 2013), debería estudiarse más detalladamente la influencia de los cambios climáticos interanuales en los patrones de dispersión del polen y otros factores que influencian el éxito reproductivo masculino y femenino, como la fecundidad masculina y la distancia entre individuos (Dow y Ashley 1998, Burczyk et al. 2004, Torimaru et al. 2012).

Nothofagus alpina, como otras especies anemófilas, produce una gran cantidad de polen, lo que se interpreta como una estrategia reproductiva para compensar la imprecisión del viento como agente polinizador. Algunos estudios sobre producción polínica en diferentes especies vegetales, tanto en anemófilas (Reddi y Reddi 1986, Prieto-Baena et al. 2003, Gómez-Casero et al. 2004) como en entomófilas, comprueban que, por lo general, existe una mayor producción de polen en las especies polinizadas por el viento (Tormo et al. 1996). En conclusión, la cantidad de polen producido no sería una limitante en la producción de semillas en el huerto Huillilemu y según García et al. (2015), el polen presenta valores altos de viabilidad, por lo que la viabilidad del polen tampoco se debe considerar como un factor limitante.

En la literatura no existen datos sobre la producción de polen por flor en especies del género Nothofagus, pero en especies emparentadas del orden Fagales se ha documentado para Quercus coccifera L. la cantidad de 7.355 granos de polen por estambre en el año 2000 y 6.804 en el 2001 y para Quercus ilex (Lam.) Schwartz ex T. Morais. y Quercus suber L. alrededor de 3.500 granos de polen por estambre en los mismos periodos de estudio (Gómez- Casero et al. 2004). Estos datos no distan mucho de lo calculado para $N$. alpina., entre 1,900 y 6,480 granos de polen por antera, considerando que cada estambre tiene una antera.

En este trabajo, se ha observado que el número de granos de polen por antera determina el contenido de granos de polen por flor. Esto es relevante por dos motivos: 1) la relación funcional entre polen/antera y polen/flor es independiente del clon de $N$. alpina y del año de evaluación (esto refleja una relación funcional muy robusta), 2) no se observa ningún tipo de relación entre en número de ante- ras/flor y la cantidad de polen/flor, lo que sugiere que la cantidad de polen/antera es más determinante que el número de anteras/flor en el contenido final de polen/flor.

La determinación de los factores relacionados a la variabilidad interanual en la producción de polen, constituirá un conocimiento valioso para optimizar la producción de semillas y desarrollo de nuevos genotipos en los programas de mejoramiento genético.

\section{CONCLUSIONES}

La baja producción de semillas en condiciones de polinización abierta parece estar relacionada con la insuficiente llegada de polen a los estigmas, pues la formación de semillas viables después de polinización manual fue notablemente mayor.

La producción de polen por flor, dentro de una misma temporada, es diferente entre los árboles analizados, igualmente para un mismo individuo en los diferentes años de evaluación, lo que sugiere la existencia de diferencias genéticas entre individuos y un importante efecto de las diferentes condiciones climáticas durante los años en que se realizó el estudio.

La cantidad de polen contenido en las flores de N. alpina varía en relación constante con los granos de polen en las anteras, a pesar que la cantidad de polen por flor no muestra la existencia de una relación funcional con el número de anteras. Esto sugiere que la producción final de polen es determinada dominantemente por la reducción del contenido de granos de polen por antera y no por la modificación del número de anteras por flor.

\section{REFERENCIAS}

Ashman T, T Knight, J Steets, P Amarasekare, M Burd, D Campbell, M Dudash, M Johnston, J Mazer, R Mitchell, M Morgan, W Wilson. 2004. Pollen limitation of plant reproduction: ecological and evolutionary causes and consequences. Ecology 85: 2408-2421.

Pearse I, W Koenig, K Funk, M Pesendorfer. 2015. Pollen limitation and flower abortion in a wind-pollinated, masting tree. Ecology 96: 587-593.

Allison T. 1990. Pollen production and plant density affect pollination and seed production in Taxus canadensis. Ecology 71: 516-522.

Bierzychudek P. 1981. Pollinator limitation of plant reproductive effort. American Naturalist 117: 838-840.

Burczyk J, A Lewandowski, W Chalupka. 2004. Local pollen dispersal and distant gene flow in Norway spruce (Picea abies [L.] Karst.). Forest Ecology and Management 197: 39-48.

Burley N, Willson MF. 1983. Mate choice in plants. Princeton, New Jersey, USA. Princeton University Press. 264 p.

Donoso P y D Soto. 2010. Plantaciones con especies nativas en el centro-sur de Chile: experiencias, desafíos y oportunidades. Bosque Nativo 47: 10-17.

Dow B, M Ashley. 1998. Factors influencing male mating success in bur oak, Quercus macrocarpa. New Forests 15: 161-180. 
García L, F Droppelmann, M Rivero. 2013. Morfología y fenología floral de Nothofagus alpina (Nothofagaceae) en un huerto semillero clonal en la región de Los Ríos, Chile. Bosque 34(2): 221-231.

García L. 2014. Biología reproductiva de Nothofagus alpina Oerts. Tesis Doctorado en Ciencias Forestales. Universidad Austral de Chile. Valdivia, Chile. 117 pp. Consultado 01 de oct. 2016. Disponible en http://repositorio.educacionsuperior.gob.ec/bitstream/28000/1353/1/T-SENESCYT-00479. pdf.

García L, M Rivero, F Droppelmann. 2015. Descripción morfológica y viabilidad del polen de Nothofagus alpina (Nothofagaceae). Bosque 36(3): 487-496.

Gómez-Casero M, C Gálan, E Domínguez-Vilches. 2007. Flowering phenology of Mediterranean Quercus species in different locations (Córdoba, SW Iberian Peninsula). Acta Botánica Malacitana 32: 127-146.

Gómez-Casero M, P J Hidalgo, H García-Mozo, E Domínguez, C Galán. 2004. Pollen biology in four Mediterranean Quercus species. Grana 43: 1, 22-30.
Piotrowska K. 2008. Pollen production in selected species of anemophilous plants. Acta Agrobotanica 61 (1): 41-52

Prieto- Baena J, P Hidalgo, E Domínguez, C Galán. 2003. Pollen production in the Poaceae family. Grana 42: 153-160.

Reddi S, N Reddi. 1986. Pollen production in some anemophilous angiosperms. Grana 25: 55-61.

Ruiz-Zapata T and M Arroyo. 1978. Plant reproductive ecology of a secondary deciduos forest in Venezuela. Biotropica 10: 221-230.

Shivanna K, N Rangaswamy. 1992. Pollen biology: a laboratory manual. New York, USA. Springer. 118 p.

Servicio de Meteorología de Chile. 2016. Informes agroclimáticos, Región de los Ríos. Consultado 01 mar. 2016. Disponible en www.meteochile.cl

Tormo R, A Muñoz, I Silva, F Gallardo. 1996. Pollen production in anemophilous trees. Grana 20: 38-46.

Torimaru T, U Wennstro, D Lindgren, X Wang. 2012. Effects of male fecundity, interindividual distance and anisotropic pollen dispersal on mating success in a Scots pine (Pinus sylvestris) seed orchard. Heredity 108: 312-321.

Recibido: 12.10 .16

Aceptado: 05.01.17 
